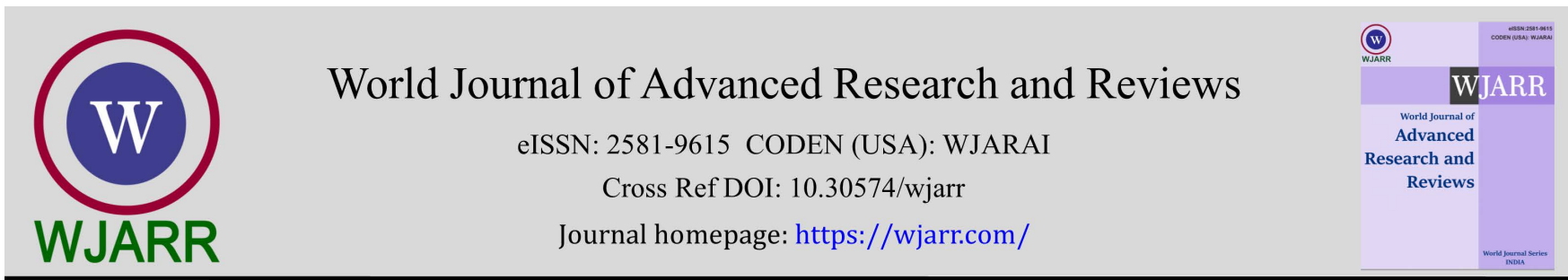

(RESEARCH ARTICLE)

Check for updates

\title{
Ex vivo liver resection for liver tumors: Last resort when conventional technique is not applicable
}

\author{
Danilo Coco ${ }^{1,{ }^{*}}$ and Silvana Leanza ${ }^{2}$ \\ ${ }^{1}$ Department of General Surgery, Ospedali Riuniti Marche Nord, Pesaro, Italy. \\ 2 Department of General Surgery, Carlo Urbani Hospital, Jesi (Ancona), Italy.
}

World Journal of Advanced Research and Reviews, 2021, 10(02), 006-009

Publication history: Received on 24 February 2021; revised on 27 March 2021; accepted on 30 March 2021

Article DOI: https://doi.org/10.30574/wjarr.2021.10.2.0091

\begin{abstract}
Purpose: The treatment of hepatic neoplasms has undergone, in recent years, various evolution of the surgical technique and extension to the indication for resection. Many patients with liver tumors cannot benefit from resection due to the difficulty of the anatomical site of the lesion.Of these patients, only some can benefit from ex vivo hepatic resection, which consists of a complete hepatectomy, bench tumor resection and self-transplant.
\end{abstract}

Materials and methods: We have retrospectively evaluated PUBMED databases. Studies was evaluated from 2010 to 2020.0nly very few studies analyzed "Ex situ liver resection", "Extracorporeal liver resection", "Liver autotransplantation".

Conclusion: Ex vivo liver resection and autotransplantation is fesible in very few patients with unresectable hepatic tumor fit for surgery. R0 resection accounts about $60 \%-90 \%$ but outcomes are less satisfactory due to high complications rate of about $25 \%$ and low survival in 3 years.

Keywords: Ex situ liver resection; Extracorporeal liver resection; Liver auto-transplantation

\section{Introduction}

Primary liver malingnancies such as HepatoCellular Carcinoma(HCC), CholangioCarcinoma(CC) and secondary tumors such as Colo-Rectal Liver Metastases(CRLM) are the most common neoplasms in the liver. R0 resection can achieve 30\%$50 \%$ of 5 year median survival while R1-2 resection achieves only 7\%.(1). However, some hepatic tumors are considered unresectable because of large size or for involving Retro-hepatic Inferior Vena Cava (RIHV) or Hepato-caval Confluence(HC) or because liver can safely tolerate total vascular occlusion for only about 60-90 min (2). By Ex vivo Liver Resection and Autotransplantation (EVLRAT) we mean the removal of the liver from the abdominal cavity, the subsequent resection of the tumor lesion on the table in cold ischemia and the subsequent reimplantation of the same organ deprived of the tumor. As early as 1988, Pichlmayr (3) and colleagues made the first attempt. This technique allows the surgeon to have yet another liver resection technique, to be able to access sections of the liver that are difficult to access, to be able to resect the neoplasm better, to operate in a bloodless operating field, to reconstruct the vessels and to avoid a hot ischemia harmful to the liver (4). However, this technique is not used frequently due to the fact that advanced tumors near or involving vascular structures have a poor prognosis, due to the fact that it is an extremely complex technique and, last but not least, that it has a high morbidity and mortality $(2,4,5,6,7,8,9)$. Actually, does not exit an well-stabilish guidelines.The ideal fit patient is the patient suffers of benign tumor or low-grade tumor, huge tumor, tumor near vital structures such as hepatic artery or vena cava or hepatic veins and a patient who can achieves long-term survival.

\footnotetext{
${ }^{*}$ Corresponding author: Danilo Coco MD

Department of General Surgery, Ospedali Riuniti Marche Nord, Pesaro, (Italy)..

Copyright $(2021$ Author(s) retain the copyright of this article. This article is published under the terms of the Creative Commons Attribution Liscense 4.0.
} 


\section{Methods}

We have retrospectively evaluated PUBMED databases. Studies was evaluated from 2000 to 2020.0 nly very few studies analyzed a "Ex vivo liver resection". Two independent research DC and SL performed the review. The search terms were identified with medical subject heading (MeSH). Research inclusion criteria were "Ex vivo liver resection ","Ex situ liver resection","extracorporeal liver resection","liver autotransplantation". The outcomes were complete were indications,operative time,blood loss, morbidity, and mortality.We exclude all manuscript abstract and non-english manuscript. After rejecting review articles and repetitive reports, the relevant literature included 9 manuscripts.

\section{Results}

Zawistowski et al.(10) analyzed fifty-three studies with total 244 patients through a Meta-analysis.The study revealed R0 resection rate was achived in $93 \%$ of cases with major surgical complications of $25 \%$, a 30 -day mortality of $10 \%, 90$ day mortality rate was $12 \%$ for malignant tumors versus $8 \%$ in benign tumors and a 1-year survival of $79 \%$.In particular post-operative 1 year survival was $65 \%$ for malignant tumors and $90 \%$ for benign tumors.Cheng et al.(11) describes EVLRAT in 43 patients suffering from HepatoCarcinoma and Cholangiocarcinoma (HCC/CCC). They demonstrated some notes: duration operative time 8 hours, blood loss about $1500 \mathrm{cc}$, anhepatic time high of 250 minutes, mortality rate of 20\% 9/43 patients.Aji et al.(12)analyzed 69 cases of EXVLRAT for echinococcosis demonstrated similar operative time and blood loss but a better 30 day and 90 day mortality respectively of $7 \%$ and $11 \%$ and 1 year survival rate of $87 \%$ of patients, showing a better prognosis and utility of this techniques for non-malignant liver tumors.Applying EVLRAT for alveolar Echinococcosis,Beldi et al.(13) noted that the survival rate in the group of autotransplantation was greater than those who underwent allotransplantation.Boggi et al.(14) applied the ex situ technique repair in a patient with severe liver trauma in such an emergency situation.Hemming et al.Yang et al.and Mo et al. $(15,16,17)$ used a temporary portacaval shunt avoiding an extracorporeal veno-venous bypass.Sugimachi K et al.(18) described the resection of a huge HCC of $18 \mathrm{~cm}$ involved IVC and RHC in a young patient.Oldhafer et al.(19) described 22 cases of EVLRAT 22 colorectal metastases $(n=9)$, leiomyosarcoma $(n=3)$, hepatocellular carcinoma $(n=2)$, cholangiocellular carcinoma $(n$ $=2)$, Klatskin tumors $(n=4)$, focal nodular hyperplasia $(n=2)$ with in-mortality rate of 6/22.Lodge et al. (20) EVRLR for 4 cases of colorectal metastases with1/4 in mortality while Lechaux and Chui et al. $(21,22)$ demonstrated no mortality aftyer EVLRAT after hilar cholangiocarcinoma resections.Ikegami et al.(23) published a case of resection of hemangiomas with no mortality.

\section{Discussion}

Liver resections have had, over time, a rapid improvement in technique, technical means and survival. However, some primary or secondary tumors of the liver are difficult to remove due to their location and size. These include those involving the infrahepatic vena cava and those of the hepatocaval junction. Ex Vivo Liver Resection and AutoTransplantation (EVLRAT) is a challengig procedure in patients with hepatic tumors in whom is not possible classical liver resection.This technique consists in total hepatectomy,liver back-table resection and then autotransplantation.This procedure seems to achive an 1 year survival rate of $82 \%$, R0 resection in $98 \%$ of cases but has many complications, high peri-operative mortality about $9 \%$ expecially for EVRAT for malignant tumors.As reviwed in the literature,it can be applied for malignant tumors such as HCC,CC,CRLM but also for benign diseses such as Alveolar Echinococcosis (AE) or Hemangiomas(H).EVLRAT has many advantages:the back-table resection facilitates the chances of obtaining R0 resection;gives the chances to reconstruction of blood vessels; it is not a time-consuming technique respect to conventional surgery where warm ischemia time is present; autotransplantation avoid problems of transplant surgery. $(5,24,25,26,27,28)$. EVLRAT permits to use radiotherapy in ex situ liver avoiding radiotherapy complications in abdomen(7) and it seems to have less cost comparing it with allotransplantation(28). EVLRAT needs of trasplantation team, complex anesthesia and a multidisciplinary approach. It is reserved to fit for surgery patients with non-cicirrhotic liver,normal liver functions and unresectable tumors with conventionally resection and it represents the last chance when other types of techniques failure.Correct indications are:large tumors, risk hemorrrhage of in situ resection,risk of liver failure for a prologned warm ischemia, tumors in difficult anatomic locations such as infiltration of retro-hepatic inferior vena cava or hepatic vein or hepato-caval conflunence or large tumors near biliary structures $(2,5,6,7,8)$. Analyzing the review of literature results, we can notice that: segment resction were from 1 segment to six segments:operative time was between $7 \mathrm{~h}-19 \mathrm{~h}$; anhepatic phase had a time between 168384 min and the main complications were bleeding,liver failure and portal thrombosis. 


\section{Conclusion}

Ex vivo liver resection and autotransplantation (EVLRAT) is feasible but very complex technique.It can apply in few patients with unresectable hepatic tumor fit for surgery, benign tumor or malignant low-grade tumor with long term survival with R0 resection about $60 \%-90 \%$ but outcomes are less satisfactory due to high complications rate of about $25 \%$ and low survival in 3 years. EVLRAT may offers a last resort when conventional technique is not applicable $(2,4,5,6,7,8,9)$. In patients with no curative prospect and a life expectancy, this procedure may improve curative surgery and the patient's quality of life $(29,30)$.

\section{Compliance with ethical standards}

\section{Acknowledgments}

The authors thank all the general surgery staff for their cooperation. The authors have no conflict of interest and no financial issues to disclose. All the authors read and approved the paper.

\section{Disclosure of conflict of interest}

The authors declare no competing interests.

\section{References}

[1] Simmonds PC, Primrose JN, Colquitt JL, Garden OJ, Poston GJ, Rees M. Surgical resection of hepatic metastases from colorectal cancer: a systematic review of published studies. Br J Cancer. 2006; 94: 982-999.

[2] Hemming AW, Reed AI, Fujita S. Ex vivo extended left hepatectomy with caval preservation, temporary portacaval shunt, and reconstruction of the right hepatic vein outflow using a reversed portal vein bifurcation graft. J Hepatobiliary Pancreat Surg. 2006; 13: 525-529.

[3] Pichlmayr R, Bretschneider H, Kirchner E, Ringe B, Lamesch P, Gubernatis G, et al. Ex situ operation on the liver. A new possibility in liver surgery. Langenbecks Arch Chir. 1988; 373: 122-126.

[4] Regina Hwang, Peter Liou, Tomoaki Kato. Ex vivo liver resection and autotransplantation: An emerging option in selected indications, journal of Hepatology. 2018; 69(5): P1002-1003.

[5] Sun ZQ, Ding Y, Jiang YC, et al. Ex situ hepatectomy and liver autotransplantation for a treating giant solitary fibrous tumor: A case report. Oncol Lett. 2019; 17: 1042e1052.

[6] Wang H, Liu Q, Wang Z, Zhang F, Li X, Wang X. Clinical outcomes of ex vivo liver resection and liver autotransplantation for hepatic alveolar echinococcosis. J Huazhong Univ Sci Technol Med Sci. 2012; 32: $598 \mathrm{e} 600$.

[7] Buchholz BM, Pinter Carvalheiro da Silva Boteon A, Taniere P, Isaac JR, Gourevitch D, Muiesan P. Autotransplantation of the liver for ex vivo resection of intrahepatic caval leiomyosarcoma: a case report. Exp Clin Transplant. 2020; 18: 396e401.

[8] Qiu YW, Yang XW, Shen S, Huang B, Wang W. Vascular infiltration-based surgical planning in treating end-stage hepatic alveolar echinococcosis with ex vivo liver resection and autotransplantation. Surgery. 2019; 165: $889 \mathrm{e} 896$.

[9] George A, Rammohan A, Reddy SM, Rela M. Ex situ liver resection and autotransplantation for advanced cholangiocarcinoma. BMJ Case Reports. 2019; 12: e230808.

[10] Michał Zawistowskia, Joanna Nowaczyka, Michał Jakubczyk, PhDb, Piotr Domagała, MD, PhD, MBA, FEBSc, Outcomes of ex vivo liver resection and autotransplantation:A systematic review and meta-analysis, Surgery. 2020;168: 631e642.

[11] Cheng FJ, Yang ZY, Zeng J, et al. Anesthesia management of modified ex vivo liver resection and autotransplantation. Ann Transplant. 2018; 23: $274 \mathrm{e} 284$.

[12] Aji T, Dong JH, Shao YM, et al. Ex vivo liver resection and autotransplantation a alternative to allotransplantation for end-stage hepatic alveolar echinococcosis. J Hepatol. 2018; 69: 1037e1046. 
[13] Beldi G, Vuitton D, Lachenmayer A, et al. Is ex vivo liver resection and autotransplantation a valid alternative treatment for end-stage hepatic alveolar echinococcosis in Europe? J Hepatol. 2019; 70: 1030e1031.

[14] Boggi U, Vistoli F, Del Chiaro M, Signori S, et al. Extracorporeal repair and liver autotransplantation after total avulsion of hepatic veins and retrohepatic inferior vena cava injury secondary to blunt abdominal trauma. J Trauma. 2006; 60: 405-406.

[15] Hemming AW, Reed AI, Fujita S. Ex vivo extended left hepatectomy with caval preservation, temporary portacaval shunt, and reconstruction of the right hepatic vein outflow using a reversed portal vein bifurcation graft. J Hepatobiliary Pancreat Surg. 2006; 13: 525-529.

[16] Yang ZY, Lu Q, Liu XD, et al. Ex-vivo liver resection combined with partial liver autotransplantation for hepatocellular carcinoma located at critical site (in Chinese). Chin J Dig Surg. 2010; 9: 18-20.

[17] Mo YW, Wang TC, Sun ZW, et al. Extracorporeal hepatectomy and autotransplantation of residual liver for hepatic space-occupying lesion (in Chinese). Chin J Pract Surg. 2008; 28: 880-882.

[18] Sugimachi K, Shirabe K, Taketomi A, et al. Successful curative extracorporeal hepatic resection for far-advanced hepatocellular carcinoma in an adolescent patient. Liver Transplantation. 2010; 16: 685-687.

[19] Oldhafer KJ, Lang H, Schlitt HJ, Hauss J, Raab R, Klempnauer J, Pichlmayr R. Longterm experience after ex situ liver surgery. Surgery. 2000; 127: 520-527.

[20] Lodge JPA, Ammori BJ, Prasad KR, et al. Ex vivo and in situ resection of inferior vena cava with hepatectomy for colorectal metastasesAnn Surg. 2000; 4: 471-479.

[21] Lechaux D, Megevand JM, Raoul JL, et al. Ex vivo right trisegmentectomy with reconstruction of inferior vena cava and 'flop' reimplantation. J Am Coll Surg. 2002; 194: 842-845.

[22] Chui AKK, Rao AR, et al. Ex situ ex vivo liver resection, partial liver autotransplantation for advanced hilar cholangiocarcinoma: a case report. Transplant Proc. 2003; 35: 402-403.

[23] Ikegami T, Soejima Y, Taketomi A, et al. Extracorporeal hepatic resection for unresectable giant hepatic hemangiomas. Liver Transpl. 2008; 14: 115-117.

[24] Hibi T, Sapisochin G. What is transplant oncology? Surgery. 2019; 165: 281e285.

[25] Hemming AW, Reed AI, Langham Jr MR, Fujita S, Howard RJ. Combined Resection of the liver and inferior vena cava for hepatic malignancy. Ann Surg. 2004; 239: 712e719.

[26] Hemming AW, Mekeel KL, Zendejas I, Kim RD, Sicklick JK, Reed AI. Resection of the liver and inferior vena cava for hepatic malignancy. J Am Coll Surg. 2013; 217: 115e124.

[27] Gruttadauria S, Marsh JW, Bartlett DL, Gridelli B, Marcos A. Ex situ resection techniques and liver autotransplantation: last resource for otherwise unresectable malignancy. Dig Dis Sci. 2005; 50: $1829 \mathrm{e} 1835$.

[28] He YB, Bai L, Jiang Y, et al. Application of a three-dimensional reconstruction technique in liver autotransplantation for end-stage hepatic alveolar echinococcosis. J Gastrointest Surg. 2015; 19 : 1457 e1465.

[29] Peng Lei XueMing Liu ShiQi Liu Yi Lv, Ex situ Liver Resection for Unresectable Tumors, Dig Surg. 2012; 29: 140148.

[30] Yanaga K, Kishikawa K, Shimada M, Kakizoe S, Higashi H, Nishizaki T, et al. Extracorporeal hepatic resection for previously unresectable neoplasms. Surgery. 1993; 113: 637-643. 